\title{
Acceleration of alkyne metathesis in multicomponent catalytic systems by use of alternative Mo(0) sources under optimised conditions
}

Maciej Zaranek ${ }^{1 *}$ and Jakub Robaszkiewicz ${ }^{2}$

1. Centre for Advanced Technologies, Adam Mickiewicz University, Uniwersytetu Poznańskiego st. 10, 61-614 Poznań, Poland

2. Faculty of Chemistry, Adam Mickiewicz University, Uniwersytetu Poznańskiego st. 8, 61 -614 Poznań, Poland

\begin{abstract}
Alkyne metathesis in multicomponent catalytic systems, although subject to changes, is still a domain of molybdenum hexacarbonyl as a source of Mo(0). Our findings show that this coordination compound is relatively inert under metathesis conditions, which results in noticeably long induction of catalytic activity, and the kinetics of this transformation can greatly benefit from switching to more labile $\mathrm{Mo}(0)$ complexes. Several easily obtainable ones had been tested and $\left[\mathrm{Mo}(\mathrm{CO})_{3}(\mathrm{py})_{3}\right]$ has been chosen as the one exhibiting the most desired features, i.e. nearly instant catalytic activity and relative stability in the air.
\end{abstract}

Abbreviations: mes - 1,3,5-trimethylbenzene, nbd - norborna-2,5-diene; cod - cycloocta-1,5-diene; py - pyridine

Keywords: alkyne metathesis, molybdenum, silanols, multicomponent

\section{Introduction}

Over the last decade, alkyne metathesis has emerged as a viable tool for organic synthesis.[1,2] Arguably, this fact can be attributed to almost explosive research[3] in alkylidyne complexes of molybdenum[4-7], tungsten[6], and, most recently, rhenium, [8] however, the original multicomponent catalytic system is the more accessible option that still can find uses.[9-12]

Alkyne metathesis is, in principle, analogous to its better known relative - olefin metathesis. Whereas the latter is an exchange of alkylidene units of alkenes, the former constitutes a rearrangement of alkylidyne moieties of alkynes. The first report on alkyne metathesis, originally as a heterogeneous process, has been made in 1968 by Pennella, Banks, and Bailey.[13] It took further six years until Mortreux and Blanchard devised the first homogeneous catalytic system which then comprised of hexacarbonylmolybdenum(0) and resorcinol,[14] and another seven years for Wengrovius, Sancho, and Schrock to publish their findings on exceptional potency of alkylidyne complexes of W(VI) in catalysing this reaction.[15] Despite unrivalled reaction rates when using alkylidyne complexes, it was mostly the multicomponent catalytic system which was used and developed during the initial period. The reason for this state of affair could be that aforementioned Schrock's complexes are cumbersome to prepare and handle, which discouraged researchers potentially interested in alkyne metathesis. 
a)

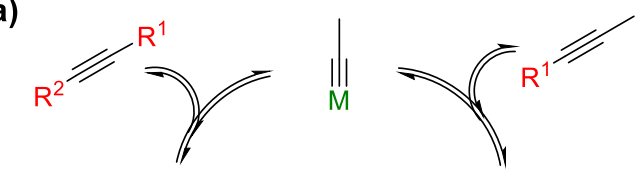<smiles>[R]C1=C([R])C(C)=[M]1CCC</smiles><smiles>[R]C1=[M]C(C)=C1C1CC([R])=C(C)C1=[W]</smiles><smiles>[R]C#CC=CC(=C)C=CC(C=CC)=CC</smiles>

b)

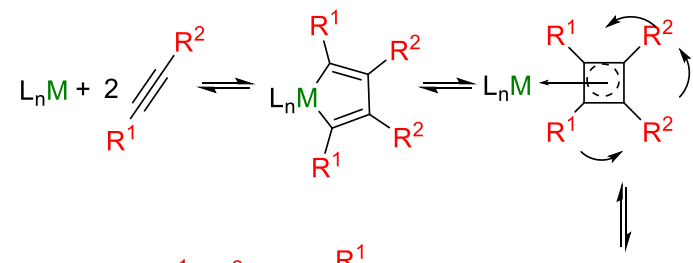

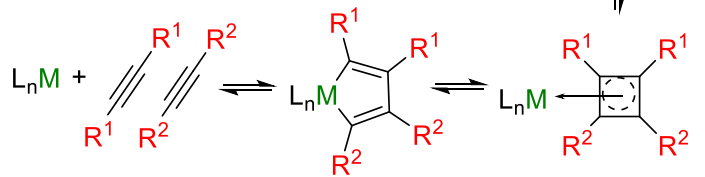

Scheme. 1. Alkyne metathesis mechanisms: a) proposed by Katz for alkylidyne catalysts; b) proposed by Mori for multicomponent catalytic systems.

Over the years, the mechanism of alkyne metathesis was explained in two different ways, depending on the kind of catalytic system being considered. Early on, Katz has proposed that alkyne metathesis occur on the way analogous to olefin metathesis, described by the Chauvin's mechanism, i.e. by [2+2] cycloaddition of alkyne to alkylidyne to form metallacyclobutadiene (Scheme 1, panel a).[16] It was not immediately clear how this proposal could apply to Mortreux's Mo(0)/phenol catalytic systems whose explanation has been proposed by Mori.[17] She has devised a mechanism in which two molecules of alkyne undergo reductive coupling with the $\operatorname{Mo}(0)$ on the way of $[1+2+2]$ cycloaddition to form a metallacyclopentadiene (Scheme 1, panel b). The latter, in turn, forms a $\eta^{4}$-cyclobutadiene complex by reductive elimination. According to Mori, it be the rotation of this ligand, its oxidative addition and subsequent cycloreversion of metallacyclopentadiene, what makes for the actual redistribution of alkylidyne moieties in alkyne reagents. Some possibilities of generation of alkylidyne complexes in multicomponent catalytic systems include formation of $\mathrm{Mo} \equiv$ Mo cluster complexes which undergo metathesis with alkynes.[18] Given that this reaction of $\left[\mathrm{Mo}(\mathrm{CO})_{6}\right]$ is rate-limited by the release of $\mathrm{CO}$, it seemed a viable idea to examine other, more labile sources of $\mathrm{Mo}(0)$. This report is a summary of such catalytic trials.

\section{Methods}

2.1. General remarks. All reactions were carried out under argon atmosphere using standard Schlenk techniques and thoroughly dried glassware. Toluene was purified by distillation over sodium/benzophenone and stored under argon over small amount of calcium hydride. Alkynes were 
dried over calcium hydride and purified by vacuum trap-to-trap distillation. Dimethyl(naphthyl)silanol was synthesised as reported previously.[19] $\left[\mathrm{Mo}(\mathrm{CO})_{3}(\mathrm{mes})\right],[20] \quad\left[\mathrm{Mo}(\mathrm{CO})_{4}(\mathrm{nbd})\right],[21]$ $\left[\mathrm{Mo}(\mathrm{CO})_{4}(\mathrm{cod})\right],[22]\left[\mathrm{Mo}(\mathrm{CO})_{3}(\mathrm{MeCN})_{3}\right],[23]\left[\mathrm{Mo}(\mathrm{CO})_{3}(\mathrm{PhCN})_{3}\right],[24]$ and $\left[\mathrm{Mo}(\mathrm{CO})_{3}(\mathrm{py})_{3}\right]$ [25] were synthesised according do known literature procedures. GC analyses were performed on a Bruker 436GC gas chromatograph with a TCD detector using decane as reference. GC-MS analyses were performed on Bruker Scion 436-GC with Bruker Scion SQ mass detector. In situ IR measurement was performed on a Mettler Toledo ReactIR ${ }^{\mathrm{TM}} 15$ system.

2.2. Alkyne metathesis. Reactions were carried out in a $25 \mathrm{~mL}$ round-bottom flask with integrated air condenser, inert gas plug with a valve, and a thread side arm equipped with a septum screw cap. The flask was evacuated and dried at approximately $200{ }^{\circ} \mathrm{C}$. After cooling to room temperature and argon re-fill, the flask was charged with silanol (typically $0.08 \mathrm{mmol})$, toluene $(1 \mathrm{~mL})$, decane $(100 \mu \mathrm{L})$, and alkyne $(1.00 \mathrm{mmol})$. A reference $\mathrm{GC}$ sample $(20 \mu \mathrm{L})$ was taken and molybdenum( $(0)$ complex (typically $0.02 \mathrm{mmol}$ ) was added. Next, reaction system was placed in an oil bath pre-heated to $120^{\circ} \mathrm{C}$. During the course of the reaction, $20 \mu \mathrm{L}$ samples of the solution were taken at specific times to determine conversion. After the reaction, a sample was analysed by GC-MS to confirm selectivity of metathesis.

\section{Results and discussion}

In our previous work, we found out that $\left[\mathrm{Mo}(\mathrm{CO})_{6}\right]$ decays completely in the course of UVactivated alkyne metathesis. To determine whether this is also true for thermally-activated systems, an experiment was performed during which in situ IR spectra of the reaction solution of degenerate metathesis of 4-octyne were recorded. The comparison of thermally and photochemically activated reactions in terms of hexacarbonylmolybdenum(0) consumption is shown in the Graph 1.

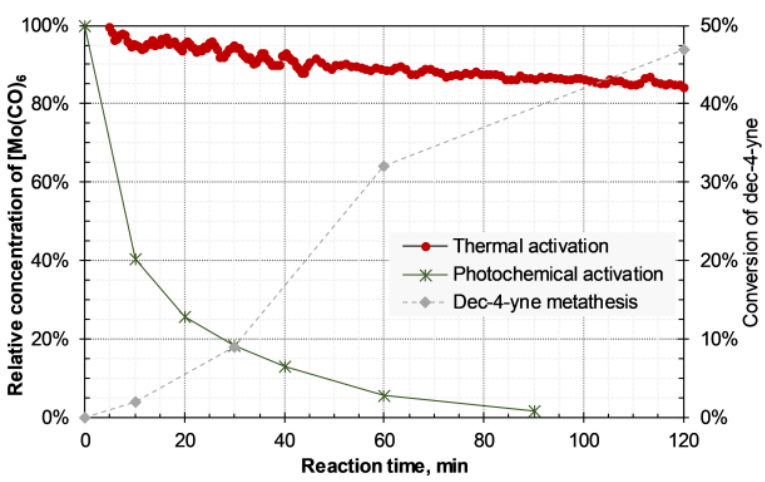

Graph 1. Comparison of $\left[\mathrm{Mo}(\mathrm{CO})_{6}\right]$ decay in photochemically and thermally activated metathesis along with a kinetic profile of dec-4-yne thermal metathesis.

The chart shows a very clear distinction between the two presented reactions. As previously described, photoactivation lead to complete conversion of $\left[\mathrm{Mo}(\mathrm{CO})_{6}\right]$ in about 90 minutes. It explains why in the case of such activation method, the final conversion of alkyne was always achieved in this time and the reaction did not progress further. On the other hand, thermal metathesis used up only a small portion of the initial amount of the complex, mostly in the first hour of reaction. It appears consistent with the fact that well-defined complexes of documented catalytic activity can usually be used in much smaller 
quantities while still providing very good reaction rates and suggests that generation of such active catalysts is preferred under thermal activation. On the premise of this observation, a hypothesis was conceived that more labile complexes of general formulae $\left[\mathrm{Mo}(\mathrm{CO})_{3} \mathrm{~L}_{3}\right]$ and $\left[\mathrm{Mo}(\mathrm{CO})_{4} \mathrm{~L}^{\wedge} \mathrm{L}\right]$ would be more suitable $\operatorname{Mo}(0)$ sources for alkyne metathesis. Later we have found that this concept had already been only briefly mentioned and not studied more systematically or conclusively.[26,27]

In the first stage of the research, three complexes of molybdenum(0) with $C$-donor $\pi$ ligands were synthesised: $\left[\mathrm{Mo}(\mathrm{CO})_{3}(\mathrm{mes})\right] \mathbf{1},\left[\mathrm{Mo}(\mathrm{CO})_{4}(\mathrm{nbd})\right] \mathbf{2}$, and $\left[\mathrm{Mo}(\mathrm{CO})_{4}(\mathrm{cod})\right] \mathbf{3}$. Each of them was used under conditions established as optimal for $\left[\mathrm{Mo}(\mathrm{CO})_{6}\right]$ in the previous study with dimethyl(naphthyl)silanol as activator.[19] The reactions were carried out in boiling toluene in $1 \mathrm{M}$ concentrations of dec-4-yne.
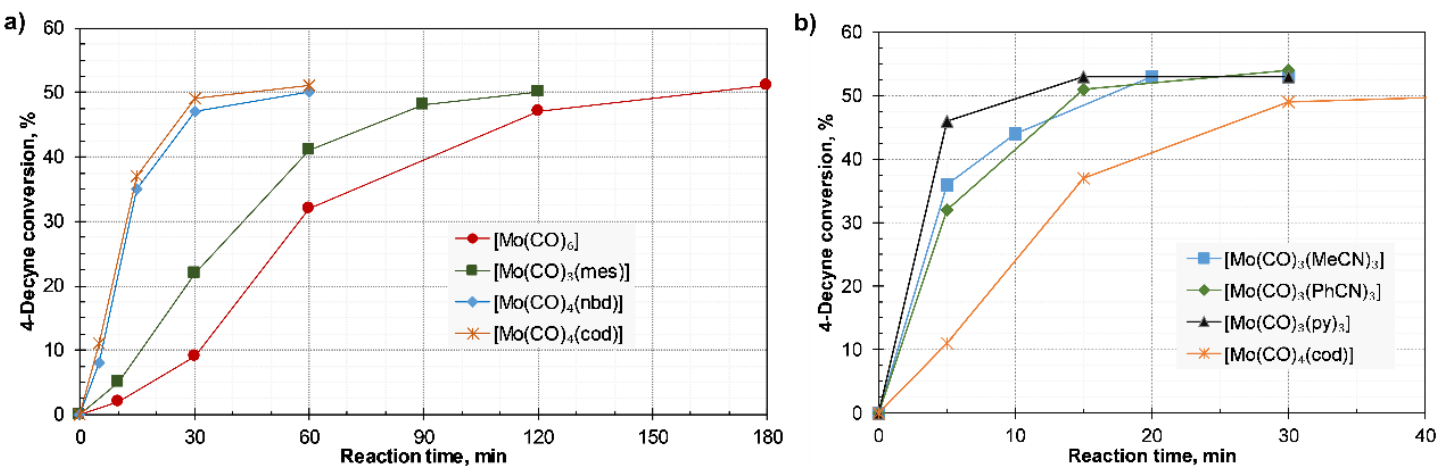

Graph 2. Kinetic profiles of dec-4-yne metathesis catalysed by $\operatorname{Mo}(0)$ complexes (note the time scale difference): a) $C$-donor $\pi$ ligands compared to $\left[\mathrm{Mo}(\mathrm{CO})_{6}\right]$; b) $N$-donor ligands compared to

$\left[\mathrm{Mo}(\mathrm{CO})_{4}(\mathrm{cod})\right]$. Conditions: toluene, reflux, $1 \mathrm{~mL}$ per $1 \mathrm{mmol}$ of dec-4-yne; activator: dimethyl(naphthyl)silanol; $[\mathrm{C} \equiv \mathrm{C}]:[\mathrm{Mo}]:[\mathrm{SiOH}]=1: 0.02: 0.08$.

As shown in Graph 2, panel a, out of $\mathbf{1}, \mathbf{2}$, and $\mathbf{3}$, the latter two allowed for visible reduction of the induction period at the beginning of reaction, leading to significant increase in the reaction rate. The use of complex 1, while still being better than $\left[\mathrm{Mo}(\mathrm{CO})_{6}\right]$, turned out to also be accompanied by an activity induction period. The initial hypothesis was most confirmed by the fact that the use and $\mathbf{2}$ and 3 led metathesis to reach equilibrium in less than 60 minutes without any other changes to the reaction system. Next, three $\mathrm{N}$-donor ligand complexes of $\mathrm{Mo}(0)$ were synthesised: $\left[\mathrm{Mo}(\mathrm{CO})_{3}(\mathrm{MeCN})_{3}\right] \mathbf{4}$, $\left[\mathrm{Mo}(\mathrm{CO})_{3}(\mathrm{PhCN})_{3}\right] 5$, and $\left[\mathrm{Mo}(\mathrm{CO})_{3}(\mathrm{py})_{3}\right]$ 6. The rationale behind the choice of such complexes was that they should be even more labile and, if it is true that the rate of metathesis is limited mostly by the initial dissociation of $\mathrm{CO}$ to allow for formation of active species, they should perform better than the former set of precatalysts. The panel b of Graph 2 shows the performance of $\mathbf{4 , 5}$, and $\mathbf{6}$ compared to previously-best 3 . All three of them greatly improved metathesis rate, leading to equilibrium in less than 20 minutes, with 6 approaching it in as little as 5 minutes. This good performance of nitrile complexes comes with a price of general instability towards oxygen and moisture. Complex $\mathbf{4}$ decomposes visibly in the air in less than $\mathbf{5}$ minutes, $\mathbf{5}$ is more stable, allowing for careful weighing and transfer under atmospheric conditions, however, both of them require refrigerated storage under argon and without light exposition. Counterintuitively, 6 turned out to be not only the most active precatalyst, but also the most stable of $\mathrm{N}$-donor ligand complexes. It does not decompose visibly in the air over 15 minutes, 
which makes it suitable for casual use. These properties made for choosing $\left[\mathrm{Mo}(\mathrm{CO})_{3}(\mathrm{py})_{3}\right]$ for further trials.

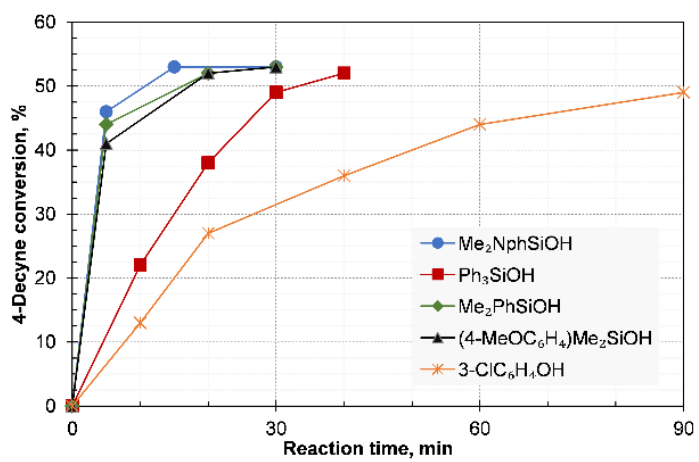

Graph 3. Kinetic profiles of dec-4-yne metathesis using precatalyst 6 at $110{ }^{\circ} \mathrm{C}$ with different activators. Conditions: toluene, reflux, $1 \mathrm{~mL}$ per $1 \mathrm{mmol}$ of dec-4-yne; $[\mathrm{C} \equiv \mathrm{C}]:[\mathrm{Mo}]:[\mathrm{SiOH}]=$ $1: 0.02: 0.08$

To examine the influence of other activators on dec-4-yne metathesis with $\mathbf{6}$, triphenylsilanol, dimethyl(phenyl)silanol, dimethyl(4-methoxyphenyl)silanol, and 3-chlorophenol were used as shown in the Graph 3. All of these were able activate the reaction, however with visibly different rates. Dimethyl(aryl)silanols gave very similar results and the use of $\mathrm{Me}_{2} \mathrm{NphSiOH}$ was again only a choice of convenience, owing to its solid state and stability. Triphenylsilanol was visibly less effective, and 3chlorophenol led to equilibrium only after more than 90 minutes of heating. A proposed explanation of the effectiveness of dimethyl(aryl)silanols is that the formation of an active complex needs ligands to be able to coordinate quickly after dissociation of pyridine. Triphenylsilanol, which is a common ligand in active well-defined complexes, is bulky and cannot approach Mo centre quick enough. This, in turn, can lead to Mo clusterisation and decrease in effective catalyst concentration. On the other hand, the other silanols combine the required feature of having an aryl substituent with being considerably less bulky.

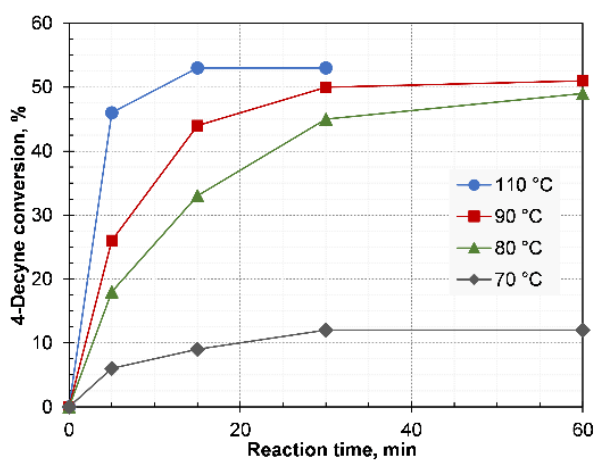

Graph 4. Kinetic profiles of dec-4-yne metathesis using precatalyst 6 at various temperatures with $\mathrm{Me}_{2} \mathrm{NphSiOH}$ activator. Conditions: toluene, $1 \mathrm{~mL}$ per $1 \mathrm{mmol}$ of dec-4-yne; $[\mathrm{C} \equiv \mathrm{C}]:[\mathrm{Mo}]:[\mathrm{SiOH}]=$ $1: 0.02: 0.08$ 
Having determined the most effective catalytic system composition, we attempted at decreasing the reaction temperature (Graph 4). These efforts were only moderately successful. In general, decrease in reaction temperature led to visible decrease in reaction rate. Only the reaction in refluxing toluene reached the equilibrium in less than 30 minutes. At $70{ }^{\circ} \mathrm{C}$ the reaction ceased after reaching $12 \%$ of dec4-yne conversion and a precipitate formed.

The catalytic system comprising $\left[\mathrm{Mo}(\mathrm{CO})_{3}(\mathrm{py})_{3}\right]$ and $\mathrm{Me}_{2} \mathrm{NphSiOH}$ was also applied to metathesis of 1-phenylprop-1-yne, 1-cyclopropyloct-1-yne, and methyl 2-nonynoate. The first of these has been fully converted after 60 minutes only when $20 \%$ of the silanol was used, whereas in the same conditions but with $\left[\mathrm{Mo}(\mathrm{CO})_{6}\right]$ conversion of 1-phenylprop-1-yne reached only $79 \%$ after 120 minutes.. It still remains unknown what is the difference between the role of activator in metathesis of aromatic and aliphatic alkynes. Metathesis of 1-cyclopropyloct-1-yne reached equilibrium in 5 minutes. The reaction of methyl 2-nonynoate required more activator to proceed. In a typical reaction system, 35\% conversion has been achieved in 15 minutes, but it has not gone beyond 38\%, even after 60 minutes. However, it is worth noting that metathesis of carbonyl compounds in $\left[\mathrm{Mo}(\mathrm{CO})_{6}\right]$-based catalytic system was not successful at all.

Concluding the report, we have established that labile complexes of $\operatorname{Mo}(0)$, especially tricarbonyltris(pyridine)molybdenum(0), are superior metal sources in alkyne metathesis compared to hexacarbonylmolybdenum(0), especially when accompanied by dimethyl(aryl)silanols. It was possible

to reduce the time needed to reach the equilibrium of aliphatic alkynes' metathesis by a factor of almost 20, from 180 to less than 10 minutes, and metathesis of an aromatic alkyne also proceeded visibly faster than when using $\left[\mathrm{Mo}(\mathrm{CO})_{6}\right]$. It is an improvement which can give a second life to the almost forgotten multicomponent catalytic system.

\section{Acknowledgements}

This work was financially supported by the National Science Centre (Poland), grant № UMO2017/25/N/ST5/00193. MZ gratefully acknowledges the Foundation for Polish Science (FNP) START grant № 96.2020. The authors would like to thank Rafał Januszewski for help with IR measurement.

\section{Conflict of interest}

The authors declare no conflict of intertest.

\section{References}

[1] A. Fürstner, The Ascent of Alkyne Metathesis to Strategy-Level Status, J. Am. Chem. Soc. 143 (2021) 15538-15555. doi:10.1021/jacs.1c08040.

[2] A. Fürstner, Alkyne Metathesis on the Rise, Angew. Chemie Int. Ed. 52 (2013) 2794-2819. doi:10.1002/anie.201204513.

[3] H. Ehrhorn, M. Tamm, Well-Defined Alkyne Metathesis Catalysts: Developments and Recent Applications, Chem. - A Eur. J. 25 (2018) chem.201804511. doi:10.1002/chem.201804511.

[4] J. Groos, P.M. Hauser, M. Koy, W. Frey, M.R. Buchmeiser, Highly Reactive Cationic Molybdenum Alkylidyne N -Heterocyclic Carbene Catalysts for Alkyne Metathesis, Organometallics. (2021) acs.organomet.1c00175. doi:10.1021/acs.organomet.1c00175.

[5] J. Hillenbrand, J.N. Korber, M. Leutzsch, N. Nöthling, A. Fürstner, Canopy Catalysts for Alkyne Metathesis: Investigations into a Bimolecular Decomposition Pathway and the Stability 
of the Podand Cap, Chem. - A Eur. J. (2021) 1-10. doi:10.1002/chem.202102080.

[6] H. Ehrhorn, D. Bockfeld, M. Freytag, T. Bannenberg, C.E. Kefalidis, L. Maron, M. Tamm, Studies on Molybdena- and Tungstenacyclobutadiene Complexes Supported by Fluoroalkoxy Ligands as Intermediates of Alkyne Metathesis, Organometallics. 38 (2019) 1627-1639. doi:10.1021/acs.organomet.9b00068.

[7] D.P. Estes, C. Bittner, ??scar ??rias, M. Casey, A. Fedorov, M. Tamm, C. Cop??ret, Alkyne Metathesis with Silica-Supported and Molecular Catalysts at Parts-per-Million Loadings, Angew. Chemie - Int. Ed. 55 (2016) 13960-13964. doi:10.1002/anie.201605129.

[8] M. Cui, W. Bai, H.H.Y. Sung, I.D. Williams, G. Jia, Robust Alkyne Metathesis Catalyzed by Air Stable d 2 Re(V) Alkylidyne Complexes, J. Am. Chem. Soc. 142 (2020) 13339-13344. doi:10.1021/jacs.0c06581.

[9] H. Yang, Y. Jin, Y. Du, W. Zhang, Application of alkyne metathesis in polymer synthesis, J. Mater. Chem. A. 2 (2014) 5986-5993. doi:10.1039/c3ta14227b.

[10] Y. Jin, Q. Wang, P. Taynton, W. Zhang, Dynamic covalent chemistry approaches toward macrocycles, molecular cages, and polymers, Acc. Chem. Res. 47 (2014) 1575-1586. doi:10.1021/ar500037v.

[11] W. Zhang, J.S. Moore, Shape-persistent macrocycles: Structures and synthetic approaches from arylene and ethynylene building blocks, Angew. Chemie - Int. Ed. 45 (2006) 4416-4439. doi:10.1002/anie.200503988.

[12] Q. Wang, C. Zhang, B.C. Noll, H. Long, Y. Jin, W. Zhang, A tetrameric cage with $\mathrm{D}<$ inf $>2 \mathrm{~h}</$ inf $>$ symmetry through alkyne metathesis, Angew. Chemie - Int. Ed. 53 (2014) 10663-10667. doi:10.1002/anie.201404880.

[13] F. Pennella, R.L. Banks, G.C. Bailey, Disproportionation of alkynes, Chem. Commun. (1968) 1548. doi:10.1039/c19680001548.

[14] A. Mortreux, M. Blanchard, Metathesis of alkynes by a molybdenum hexacarbonyl-resorcinol catalyst, J. Chem. Soc., Chem. Commun. (1974) 786-787. doi:10.1039/C39740000786.

[15] J.H. Wengrovius, J. Sancho, R.R. Schrock, Metathesis of acetylenes by tungsten(VI)alkylidyne complexes, J. Am. Chem. Soc. 103 (1981) 3932-3934. doi:10.1021/ja00403a058.

[16] T.J. Katz, J. McGinnis, Mechanism of the olefin metathesis reaction, J. Am. Chem. Soc. 97 (1975) 1592-1594. doi:10.1021/ja00839a063.

[17] M. Nishida, H. Shiga, M. Mori, [2+2+2] Cocyclization Using [Mo(CO) 6 - p -ClPhOH], J. Org. Chem. 63 (1998) 8606-8608. doi:10.1021/jo981240o.

[18] J. Geng Lopez, M. Zaranek, P. Pawluc, R.M. Gauvin, A. Mortreux, In Situ Generation of Molybdenum-Based Catalyst for Alkyne Metathesis: Further Developments and Mechanistic Insights, Oil Gas Sci. Technol. - Rev. d'IFP Energies Nouv. 71 (2016) 20. doi:10.2516/ogst/2015046.

[19] M. Zaranek, J. Robaszkiewicz, I. Janica, R.M. Gauvin, P. Pawluć, A. Mortreux, In situ $\mathrm{Mo}(\mathrm{CO})_{6}$-based catalysts for alkyne metathesis: Silanols vs phenols as co-catalysts under thermal and photochemical activation, Catal. Commun. 138 (2020) 105944. doi:10.1016/j.catcom.2020.105944.

[20] D.E. Koshland, S.E. Myers, J.P. Chesick, The crystal structures of 1,3,5trimethylbenzenetricarbonylmolybdenum and hexamethylbenzenetricarbonylmolybdenum, Acta Crystallogr. Sect. B Struct. Crystallogr. Cryst. Chem. 33 (1977) 2013-2019. doi:10.1107/S056774087700764X.

[21] M.Y. Darensbourg, M. Pala, S.A. Houliston, K.P. Kidwell, D. Spencer, S.S. Chojnacki, J.H. Reibenspies, Stereochemical nonrigidity in heterobimetallic complexes containing the bent metallocene-thiolate fragment, Inorg. Chem. 31 (1992) 1487-1493. doi:10.1021/ic00034a032.

[22] J.P. Shupp, A.S. Kinne, H.D. Arman, Z.J. Tonzetich, Synthesis and Characterization of Molybdenum(0) and Tungsten(0) Complexes of Tetramethylthiourea: Single-Source Precursors for MoS 2 and WS 2, Organometallics. 33 (2014) 5238-5245. doi:10.1021/om500567y.

[23] D.P. Tate, W.R. Knipple, J.M. Augl, Nitrile Derivatives of Chromium Group Metal Carbonyls., Inorg. Chem. 1 (1962) 433-434. doi:10.1021/ic50002a052.

[24] D.R. Lavering, US3065250A Nitrile-metal carbonyl complexes, US3065250A, 1962. https://patents.google.com/patent/US3065250A/en (accessed October 21, 2021).

[25] S.P. Nolan, C.D. Hoff, J.T. Landrum, Synthesis and thermochemistry of $\mathrm{HMo}(\mathrm{CO}) 3 \mathrm{C} 5 \mathrm{Me}$; 
Comparison of cyclopentadienyl and pentamethylcyclopentadienyl ligands, J. Organomet. Chem. 282 (1985) 357-362. doi:10.1016/0022-328X(85)87194-1.

[26] A. Bencheick, M. Petit, A. Mortreux, F. Petit, New active and selective catalysts for homogeneous metathesis of disubstituted alkynes, J. Mol. Catal. 15 (1982) 93-101. doi:10.1016/0304-5102(82)80008-4.

[27] Lioba Kloppenburg, and David Jones, U.H.F. Bunz*, High Molecular Weight Poly(pphenyleneethynylene)s by Alkyne Metathesis Utilizing "Instant" Catalysts: A Synthetic Study, (1999). doi:10.1021/MA9900535. 